Revue Française de Civilisation Britannique

XXII-3 | 2017

Forms of Activism in the United Kingdom (Grassroots Activism, Culture, Media)

\title{
«A musician for an occasion »: Britten, compositeur engagé
}

"A Musician for an Occasion": Britten as a Committed Composer

\section{Gilles Couderc}

\section{(2) OpenEdition}

\section{Journals}

Édition électronique

URL : http://journals.openedition.org/rfcb/1443

DOI : $10.4000 /$ rfcb. 1443

ISSN : 2429-4373

Éditeur

CRECIB - Centre de recherche et d'études en civilisation britannique

Référence électronique

Gilles Couderc, « "A musician for an occasion »: Britten, compositeur engagé », Revue Française de Civilisation Britannique [En ligne], XXII-3 | 2017, mis en ligne le 05 juillet 2017, consulté le 02 mai 2019. URL : http://journals.openedition.org/rfcb/1443; DOI : 10.4000/rfcb.1443

Ce document a été généré automatiquement le 2 mai 2019.

\section{cc) (†)}

Revue française de civilisation britannique est mis à disposition selon les termes de la licence Creative Commons Attribution - Pas d'Utilisation Commerciale - Pas de Modification 4.0 International. 


\title{
«A musician for an occasion »: Britten, compositeur engagé
}

\author{
"A Musician for an Occasion ": Britten as a Committed Composer
}

\author{
Gilles Couderc
}

\section{Introduction}

I believe that the artist must be consciously a human being. He is part of society and he should not lock himself up in an ivory tower. I think he has a duty to play towards his fellow creatures. It is not only a duty; I think it's a pleasure too. I want to have my music used. I would rather-this seems perhaps a silly thing to say-but I would rather have my music used than to write masterpieces which were not used .1

Ainsi s'exprime Benjamin Britten (1913-1976) en avril 1968 dans un article sous-titré « The Moral Responsibility of the Artists toward his Fellow Man. » Il répète ici des choses qui lui tiennent à cœur, déjà exprimées dans son grand discours d'acceptation du premier Prix Aspen, The Robert O. Anderson Aspen Award in the Humanities, le 31 juillet 1964. Fondé en 1963, ce prix vise à honorer toute personne considérée comme ayant contribué le plus au progrès des humanités. L'immense succès commercial de l'enregistrement de son War Requiem de 1962 n'est pas étranger au choix de Britten comme lauréat. Salué par trois citations aux Grammy Awards et record des ventes de disques classiques en 1963 avec 250 000 exemplaires vendus, son coffret noir sur lequel se détache en blanc le titre de l'œuvre a contribué plus que tout à la célébrité mondiale de Britten et du poète Wilfred Owen dont neuf poèmes, inscrits dans la liturgie de la messe des morts, dénoncent la guerre et les fauteurs de guerre, et plaident pour l'amitié entre les peuples. Si le thème semble rabâché et convenu, l'œuvre est en phase non seulement avec le contexte historique mais aussi avec l'émergence d'une contre-culture aux États-Unis et en Europe. Le War Requiem est composé après l'échec du débarquement américain dans la Baie des Cochons en avril 1961 et la crise des missiles déployés par Khrouchtchev à Cuba en mai 1962. Le RoyaumeUni est alors engagé dans la Guerre Froide au côté des États-Unis, et vient d'acheter des missiles nucléaires Solaris. Des avions américains à l'entraînement survolent 
constamment la maison de Britten à Aldeburgh, rappelant au compositeur, membre donateur de la Campagne pour le Désarment Nucléaire, la menace d'embrasement mondial qui déclenche des manifestations anti-nucléaires en Grande-Bretagne comme à travers l'Europe. Les ruines de la vieille cathédrale de Coventry qui jouxtent la nouvelle cathédrale, dans et pour laquelle l'œuvre est créée, rappellent à tous les raisons du Blitz qui la détruisit en novembre 1940 : les usines d'armement de la ville. Elles évoquent aussi les ruines d'Hiroshima et Nagasaki entrevues dans le film d'Alain Resnais, Hiroshima mon amour, de 1959. Le War Requiem s'offre donc à la fois comme caisse de résonnance aux angoisses des contemporains de Britten, comme démonstration de son engagement pacifiste dans son siècle et comme refus d'être un compositeur enfermé dans sa tour d'ivoire, coup de patte à ses confrères sérialistes ou avant-gardistes de Darmstadt auquel il reproche leur élitisme.

Britten commence son discours d'Aspen en définissant le mot «Humanities ». Lui qui n'a jamais fréquenté l'université et dont l'orthographe reste toujours incertaine, il en évacue le sens lié à la culture classique gréco-latine pour n'en garder que ce qui le rattache à l'humain, ce que souligne la citation justifiant la récompense : «To Benjamin Britten, who, as a brilliant composer, performer, and interpreter through the music of human feelings, moods, and thoughts, has truly inspired man to understand, clarify and appreciate more fully his own nature, purpose and destiny ${ }^{2}$. "Voilà qui résume, d'une manière certes grandiloquente mais fidèle, la carrière et les idéaux du compositeur, qui se présente comme un « musicien de circonstance » au service de ses contemporains. Compositeur fécond, chef d'une troupe d'opéra et directeur d'un festival de musique et des arts auquel est convié le gratin de la musique classique, de la littérature et des arts de son temps, chef d'orchestre, génial pianiste et accompagnateur, Britten n'est pas seulement engagé à défendre son art mais un art au service de ses contemporains. On s'interrogera ici sur les origines de cet engagement, notamment son engagement pacifiste en évoquant d'abord ses années d'apprentissage dans les années Trente, son adhésion à des idées de gauche et au concept d'art-parabole avant de comprendre comment cet engagement politico-artistique se traduit dans ses œuvres au service de son pacifisme et en faveur des enfants.

\section{Apprentissage dans les années trente}

3 Lorsque le jeune Britten sort diplômé du Royal College of Music en 1935, son bagage intellectuel de petit bourgeois provincial est limité à celui acquis pendant ses années d'études secondaires au collège Gresham à Holt dans le Norfolk, de 1927 à 1931. Comme ses contemporains les poètes $\mathrm{W}$. $\mathrm{H}$. Auden et Stephen Spender qui l'y précèdent, il souffre du système des prefects et du " code d'honneur » imposé aux élèves. Auden dit plus tard qu'il lui a donné un avant-goût des sociétés totalitaires: "The best reason I have for opposing Fascism is that at school I lived in a Fascist state ${ }^{3}$ ». En 1951 Spender décrit l'école dans des termes qui rappellent le 1984 de George Orwell : «[The boys] would betray me to the spirit of the school. The deep treachery of childhood infected the whole place, like a Fascist state where you discover every neighbour to be-even if he is not aware of it himself-an agent of the interests of the police. ${ }^{4}$ » Britten, comme son ami le romancier E. M. Forster, est révolté par les châtiments corporels en vogue dans les écoles anglaises. Plus tard, la cruauté des officiers et de Claggart, le maître d'armes, et la scène du Novice fouetté au premier acte de leur opéra Billy Budd de 1951 fait écho à cette violence. Suite aux retentissants procès d'Oscar Wilde en 1895, l'homosexualité d'Auden et de Britten les conduit sans doute à 
rejeter plus tard un modèle social qui les exclut et les condamne, au mieux au silence ou à l'exil, et au pire au suicide. À Gresham, Britten fréquente des élèves issus de la grande bourgeoisie conservatrice et libérale, dont certains plus tard, par réaction au carcan conservateur, se tournent vers le socialisme, comme Auden et Spender, ou le communisme, comme son condisciple Donald Maclean, agent double qui en 1951 passe à l'URSS, en pleine Guerre Froide, avec son complice Guy Burgess.

Petit garçon sensible mais volontaire, sportif et combatif, le jeune Britten finit ses études primaires à l'école de South Lodge de Lowestoft en 1925 avec le titre prestigieux de Victor Ludorum, mais l'essai qu'il écrit contre la chasse et les chasseurs est mal reçu par ses maitres. Peut-on déjà y voir l'expression de son pacifisme? Comme le montre le film de Tony Britten, Benjamin Britten: Peace and Conflict ${ }^{5}$, la chapelle du collège Gresham que Britten fréquente est élevée en 1916 comme mémorial dédié aux 107 anciens élèves morts au front et lui rappelle constamment la saignée subie par une génération d'élèves. En 1920 Gresham devient la première école britannique à fonder un club de la Société des Nations comptant 150 membres. Né en 1913, Britten est un « bébé de la guerre ». En avril 1916 ses oreilles sont remplies du fracas du bombardement de Lowestoft, sa ville natale, par la marine allemande qui atteint une maison toute proche du domicile familial. À Gresham, sa détestation de la guerre se nourrit du spectacle de l'officer Training Corps à la parade, dont les clairons sonnent aussi bien dans le War Requiem que dans son opéra pacifiste Owen Wingrave de 1971.

Entre temps, son éducation artistique se fait avec le compositeur Franck Bridge (1879-1941) qui, avec son épouse, constitue le premier couple d'artistes qu'il rencontre et chez qui il trouve une deuxième famille, puisque il les fréquente assidûment entre 1927 et son départ pour les États-Unis en 1939. Bridge l'initie non seulement à la musique contemporaine en provenance du Continent mais aussi à l'appréciation de l'architecture religieuse et de la peinture. Il lui inculque une éthique du travail, un souci de la maitrise technique, de la sincérité dans l'expression et de la nécessité de toujours progresser dont il ne se départit jamais. Bridge perd de nombreux élèves pendant la Grande Guerre et Britten est forcé d'argumenter avec lui son rejet viscéral de la chose militaire. En 1942, au tribunal militaire chargé d'accorder à Britten le statut de "conchie», objecteur de conscience, Mrs Bridge, qui témoigne en sa faveur, rapporte le dégoût que le jeune homme ressenti quand, dans les années Trente, il vit les Horse Guards parader en grande tenue dans Hyde Park ${ }^{6}$, scène reprise dans les Interludes I et II de Owen Wingrave. La rêverie d'Owen, jeune rebelle lecteur de la Queen Mab de Shelley qu'admirait Wilfred Owen, pour qui Britten est pris de passion, est alors interrompue par la vision du massacre de ces militaires.

Britten, qui a déjà publié plusieurs œuvres, commence sa carrière de compositeur professionnel en mai 1935. Il est engagé par Alberto Cavalcanti, cinéaste brésilien, directeur du son au sein du GPO Film Unit, dirigé par le talentueux John Grierson, pour composer la première de ses musiques de film, The King's Stamp. On considère John Grierson comme l'inventeur du documentaire avec son essai de 1932, First Principles of Documentary. L'influence du calvinisme de son père et du marxisme des syndicalistes des chantiers navals de son Clydeside natal détermine sa vision de l'art, facteur de progrès social, et du rôle politique de l'artiste, messie d'une conscience sociale. Pour Grierson le cinéma est une chaire dont il se sert pour faire de la propagande ${ }^{7}$. Lorsqu'il devient directeur du GPO Film Unit en 1933, il tourne des documentaires commandés par le gouvernement britannique destinés à sensibiliser le public au monde du travail. Grierson 
s'entoure de jeunes talents qui collaborent avec enthousiasme à la création d'une esthétique nouvelle, très éloignée des règles actuelles du documentaire et de la seule représentation de faits bruts dans ce que Grierson appelle «le traitement créatif de la réalité » qui incorpore un message social très fort.

Dans l'équipe de Grierson, Britten fait la rencontre de Wynstan Hugh Auden, laquelle compte autant dans sa vie que celle de son compagnon, le ténor Peter Pears. La réputation du poète, porte-parole des artistes de gauche, est déjà considérable. Britten est fasciné par la faconde et l'intellect brillant de celui qui va ouvrir son esprit à la littérature et à l'engagement politique, et façonner indirectement sa carrière et sa conception du rôle de l'artiste ${ }^{8}$, comme l'indique une lettre à une amie de Bridge :

I know you would like W.H.A. very much. He is a startling personality -but absolutely sincere and very brilliant. He has a very wide knowledge, not only of literature, but of every branch of art, and especially of politics; this last in the direction that I can't help feeling every serious person, and artists especially must have. Strong opposition to Fascism, which of course restricts all freedom of thought 9 .

Elle accompagne l'envoi d'un livre, sans doute The Poet's Tongue, anthologie de poèmes qu'Auden publie en juillet 1935, dont l'introduction marque à jamais l'œuvre de Britten. Tout en maintenant ses distances avec le concept d'art-propagande, Auden y exprime son credo dans la fonction éducative de l'art et sa puissance morale: "But Poetry is not concerned with telling people what to do, but with extending our knowledge of good and evil, perhaps making the necessity for action more urgent and its nature more clear, but only leading us to the point where it is possible for us to make a rational and moral choice. $»^{10}$ Britten s'empare de cette notion d'art éveilleur des consciences et fait aussi sienne une autre définition d'Auden, incluse dans un essai intitulé Psychology and Art Today, publié la même année : « There must always be two kinds of art, escape art, for man needs escape as he needs food and deep sleep, and parable-art, that art which shall teach man to unlearn hatred and learn love ${ }^{11}$. »

Britten et Auden collaborent à Coal Face et Negroes de 1935, à Night Mail de 1936, qui utilisent des poèmes d'Auden. Les dons de musicien de Britten fascinent Auden, notamment son aptitude à mettre en musique la langue anglaise. De 1936 à 1942, Britten met en musique une vingtaine de poèmes d'Auden qui collabore à Our Hunting Fathers, cycle symphonique pour soprano et orchestre de 1936, sur des poèmes écrits ou choisis par Auden. Il dénonce à sa manière les dangers menaçant la démocratie en cette année terrible. Le mouvement intitulé Dance of Death (Hawking for the partridge), première des « danses macabres » de Britten, exprime la violence de ce pacifiste combattant et met en parallèle les souffrances qu'inflige l'homme aux animaux et celles qu'il impose à d'autres hommes, en l'occurrence les juifs allemands.

Auden et son ami Christopher Isherwood s'emploient à faire l'éducation de ce jeune provincial qui rencontre l'avant-garde artistique de son époque et pactise peu à peu avec son homosexualité, grâce aux deux compères, homosexuels, aussi décomplexés qu'il est possible de l'être dans une Angleterre où elle est passible de prison. Britten compose des musiques de scène pour le Left Theatre, théâtre de répertoire spécifiquement socialiste fondé en 1934, où brille Montagu Slater (1902-1956), poète et éditorialiste de gauche, qu'André Van Gyseghem décrit comme "about the only British writer of stature who was consistently writing for the new realistic theatre of social conscience and working-class interest ${ }^{12}$ .» Britten compose les musiques de scène de Stay Down Miner et Easter 1916, d'où le choix de Slater comme librettiste pour Peter Grimes en $1942^{13}$. Britten compose aussi pour le 
Group Theatre de Rupert Doone qui monte les pièces écrites par Auden et Isherwood, The Dog Beneath the Skin (1936), The Ascent of F6 (1936) et On the Frontier (1937), paraboles sociales et politiques expérimentales qui valent à Auden le titre de poète officiel de la gauche, Leurs modèles sont le Mahagonny-Songspeil (1927) et l'Opéra de quat'sous (1928) de Kurt Weill et de Brecht. L'inclusion d'airs chantés dans les pièces d'Auden, où la musique joue un grand rôle, témoigne de cette influence. La soprano Hedli Anderson, formée en Allemagne et consciente des progrès du nazisme, pour qui Britten compose ses Cabaret Songs, s'y illustre et devient pour eux l'équivalent de Lotte Lenya pour Weill.

11 Britten tente aussi de collaborer avec l'Unity Theatre issu du Workers' Theatre Movement, qui monte en 1936 Waiting for Lefty, pièce de l'américain Clifford Odets (1906-1963) créée en 1935 à New York, qui est un exemple de théâtre alternatif de gauche ${ }^{14}$. Unity s'inspire aussi des séries du Living Newspaper, théâtre-documentaire créé sous les auspices du Federal Theatre Project et du Works Progress Administration de Roosevelt, qui influence des aspects de l'écriture de $F 6$ et de On the Frontier. Les idéaux de Brecht et du Federal Theatre Project influencent aussi Britten grâce à son amitié avec le compositeur américain Aaron Copland (1900-1990), rencontré à Londres en 1938. Son langage moderniste d'une simplicité voulue, accessible à un public populaire, ses rythmes fantasques et complexes, son usage de la bitonalité, des cuivres et des percussions séduisent Britten qui l'invite chez lui à Snape ${ }^{15}$. Copland lui joue The Second Hurricane (1936), son opéra pour enfants intitulé "pièce-opéra pour lycéens ", récemment créé à New York à l'école de Henry Street dans le Lower East Side où Copland enseigne. Le livret d'Edwin Denby s'inspire de Der Jasager (Celui qui dit oui), «Lehrstück» ou fable composée par ses amis Brecht et Weill en 1930 ${ }^{16}$. L'œuvre met en scène le courage de lycéens d'une école du MidWest pour sauver un aviateur et célèbre les idéaux de liberté et de fraternité. Copland compose une musique d'amateurs, la «Gebrauchsmusik» du dogme brechtien, d'une véritable grandeur.

De l'aveu de Britten, The Second Hurricane exerce une influence prépondérante sur Paul Bunyan, sa première œuvre scénique d'un genre hybride, "opérette chorale " en deux actes et prologue sur un livret d'Auden, créée à New York en mai 1941 par The Columbia Theater Associates de l'Université Columbia, pendant le séjour américain de Britten et de Pears de 1939 à 1942. Elle adapte la légende du fabuleux bûcheron défricheur et met en garde les américains contre les dérives de leur société de consommation, leur puritanisme et leur démocratie. L'opérette s'inspire aussi des idéaux de la gauche américaine opérant dans le cadre du Works Progress Administration et du Federal Theater Project créés en 1935, qui apportent leur soutien officiel à l'expression d'une conscience de classe et à l'injection d'idées socialistes dans le divertissement de masse. Elle se situe dans le fil des œuvres non-réalistes d'Auden-Isherwood, et le regard acéré qu'il porte sur la société américaine agace prodigieusement ses critiques. Ils se demandent si Britten et Auden connaissent Mark Blitzstein (1905-1964), ami de Copland, sympathisant communiste et partisan d'un théâtre musical populaire, qui critique férocement l'Amérique capitaliste dans The Cradle Will Rock, musical allégorique de 1937 qui décrit les efforts de Larry Foreman pour fonder un syndicat dans la ville de Steeltown et sa lutte contre le méchant businessman, Mr Mister. Mélangeant les styles et abusant de jeux de mots obscurs, le livret de Bunyan est jugé sans action véritable ni force dramatique. Mal accueilli par la critique, il est abandonné par Britten après les premières représentations malgré ses projets de révisions ${ }^{17}$. Mais en juillet 1941, Britten et Pears lisent un article de leur ami E. 
M. Forster à propos du poème The Borough de George Crabbe qui les décide à rentrer au pays et à écrire un opéra, le futur Peter Grimes, créé en juin 1945.

\section{Un pacifiste combattant}

13 Le départ d'Auden et d'Isherwood pour les États-Unis en janvier 1939, avant celui de Britten et de Pears en avril 1939, est perçu comme un lâche abandon de la part de traitres, comme l'indique Isherwood à Britten en mars $1940^{18}$. Le cas d'Auden et d'Isherwood est évoqué à la Chambre des communes en juin 1940, indiquant la place qu'a pris ce groupe dans le paysage culturel du temps. La controverse atteint Britten nommément pendant l'été 1941, alors que ses partisans, notamment le musicologue et critique musical Ernest Newman (1868-1959) s'engagent dans la bataille pour Britten, «the battle of Britten », à l'occasion de la création à Londres de son Concerto pour violon en mai 1941. Britten est l'objet d'attaques dans le Musical Times et d'une longue polémique autour de la question de savoir si le meilleur service que l'artiste puisse rendre à son pays est de se battre ou de se consacrer à son art, et ses détracteurs gardent longtemps la mémoire du départ du compositeur, qui eut des conséquences inattendues sur sa vie à son retour en $1942^{19}$.

Le départ d'Auden semble d'autant plus inexplicable à leurs contemporains qu'il s'engage pour soutenir les Républicains espagnols. Britten ne va pas jusque-là. Il lui faut gagner sa vie et il estime qu'Auden serait plus utile vivant que mort. En janvier 1937, en partance pour l'Espagne comme ambulancier, Auden écrit sur la couverture de la partition de la Sinfonietta de Britten son poème Danse Macabre de 1936 qui associe la montée du fascisme au Satan jaillissant du Chaos après sa chute, dans le Paradis Perdu de Milton. Britten met le poème en musique dans le scherzo de la Ballad of Heroes op. 14 de 1939, fresque chorale dédiée aux combattants de la Guerre d'Espagne, créée pendant un festival de « Music for the People ». Autre "Dance of Death» violente, elle est encadrée par deux poèmes du communiste Randall Swingler (1909-1967) qui composent ce triptyque. Britten emprunte à Swingler son poème Across the darkened sky pour un double chœur a capella donné en 1938 sous le titre de Advance Democracy. Les sympathies pacifistes de Britten s'expriment plus tôt dans la musique pour le film de Paul Rotha, Peace of Britain de 1936, dont les trois minutes d'images d'archives et le message anti-réarmement déclenchent les foudres de la censure et la polémique. Sa Pacifist March de 1937 est écrite sur un texte du poète et dramaturge Ronald Duncan (1914-1982) pour un concert du Peace Pledge Union, organisation pacifiste fondée en 1934 à l'initiative de Dick Sheppard, chanoine de SaintPaul de Londres, qui compte Aldous Huxley et Bertrand Russel parmi ses membres. Duncan, qui devient le librettiste de l'opéra The Rape of Lucretia de 1946, écrit une brochure The Complete Pacifist et prête à Britten le livre du philosophe américain Richard Gregg (1885-1974), The Power of Non Violence, publié à Londres en 1935. Très influencé par Gandhi, Gregg y élabore une théorie de la résistance par la non-violence qui exerce une influence majeure sur Martin Luther King. Duncan est aussi associé à trois projets pacifistes de Britten restés lettre morte faute de moyens ou de temps. Après le bombardement d'Hiroshima et de Nagasaki, ils esquissent un oratorio au titre transparent, Mea Culpa, puis une autre œuvre dédiée à Gandhi, assassiné en 1948, et une dernière à Martin Luther King en 1968. L'œuvre d'envergure qui signale son engagement pacifiste est sa Sinfonia da Requiem, créée à New York en mars 1941. Il s'agit d'une commande du Japon pour célébrer son empire de 2600 ans, rejetée par ses commanditaires à cause de son contenu chrétien et de ses références à la Messe des 
Morts. Britten en fait une protestation pacifiste en même temps qu'une œuvre à la mémoire de ses parents :

I am making it just as anti-war as possible. I don't believe you can express social or political or economic theories in music but by coupling new music with well-known musical phrases, I think it's possible to get over certain ideas. [...] One's apt to get muddled discussing such things -all I am sure of is my own anti-war convictions as I write it ${ }^{20}$.

On reconnait ici la phraséologie héritée d'Auden concernant son refus de l'art propagande, et même l'image de l'agent secret qui parcourt ses premiers poèmes, conséquence de leur homosexualité, ainsi que la tactique, modelée sur celle d'Auden, que Britten utilise abondamment lorsqu'il veut faire passer un message implicite dans ses œuvres à ceux qui sont prêts à l'entendre à demi-mot. Britten, condamné à la marge, saura toujours profiter d'occasions officielles pour remettre en question le statu quo et faire entendre sa différence. Ainsi, le Billy Budd de 1951 est cruellement décrit comme The Twilight of the Sods, en référence à Oscar Wilde, à la personnalité du compositeur, de son interprète principal et du librettiste Forster, convaincu de l'homosexualité de Melville, et au triangle amoureux que forment ses trois protagonistes.

On relève aussi l'utilisation forstérienne du mot «muddled», qui indique ici l'incapacité qu'éprouve Britten à justifier son pacifisme de manière clairement argumentée, d'où la nécessité de se faire aider par le chanoine Stuart Morris, membre du PPU, pour l'obtention du statut d'objecteur de conscience en mai 1942, seule affirmation formelle de son pacifisme :

Since I believe that there is in every man the spirit of God, I cannot destroy and I feel it my duty to avoid helping to destroy as far as I am able, human life, however strongly I may disapprove of the individual's actions or thoughts. The whole of my life has been devoted to acts of creation [...] and I cannot take part in acts of destruction. [...] I believe sincerely that I can help my fellow human beings best by continuing the work I am most qualified to do by the nature of my gifts and training, i.e. the creation or propagation of music ${ }^{21}$.

Britten et Pears obtiennent le statut de « conchie » et partent en tournée pour participer à l'effort de guerre et soutenir le moral des populations civiles, mais ce statut, ainsi que leur liaison, jamais déclarée mais vécue au grand jour, leur vaut la rancune de ceux que les succès de Britten et la publicité autour de ses œuvres irritent. Selon Pears, la sourde hostilité qu'ils ressentent alors colore le livret de Peter Grimes, choisi pour la réouverture de la salle de Sadler's Wells le 7 juin 1945. Dans le personnage de Peter, ostracisé par son village car soupçonné de causer la mort de ses apprentis, on peut lire l'image de l'homosexuel condamné au suicide, mais aussi celle de l'artiste que rejette la société de son temps, alors que l'émergence du Welfare State pose la question de sa place dans la société22.

18 C'est d'ailleurs leur statut de "queer and conchie » qui mène à la rupture avec le Wells en juillet 1945, malgré le succès commercial de Grimes, et à la création en 1947 de The English Opera Group, la compagnie que Britten fonde avec ses collaborateurs les plus proches. Son but est de créer des œuvres nouvelles requérant un minimum de chanteurs et d'instrumentistes de manière à pouvoir partir en tournée à moindres frais en l'absence de soutien institutionnel, ce qui rappelle la Barraca de Lorca. Grâce à l'EOG, et aux subventions modestes accordées par le tout nouveau Arts Council, Britten peut produire des œuvres nouvelles dans le cadre du Festival de musique, des lettres et des arts qu'il fonde en 1948 dans la petite ville d'Aldeburgh où se déroule son Peter Grimes, et où il 
s'installe dès 1947. L'objectif est de faire participer la population locale à ce qui se veut un anti-Bayreuth selon ce qui rappelle fortement les idéaux du Group Theatre de Rupert Doone.

Auparavant Britten s'impose une épreuve lorsqu'il accompagne en juillet 1945 le violoniste Yehudi Menuhin en Allemagne dans une tournée de concerts destinée aux survivants des camps de Bergen-Belsen, libérés par les Britanniques, et organisés pour faire patienter ceux qui attendent de pouvoir rentrer chez eux. On se demande encore ce qui pousse Britten à effectuer cette tournée alors que des photos horrifiantes du camp, de ses prisonniers squelettiques et de l'immense charnier où finissent les mourants ont paru dans la presse en Grande-Bretagne dès avril 1945, notamment, celles du Britannique George Rodger (1908-1995) pour Life et Time ou celles du British Army Film and Photographic Unit qui, de 1941 à 1946, filme ou photographie les armées britanniques à travers le monde. Culpabilité du pacifiste en temps de guerre? Volonté de faire face à ce qui constitue l'expression la plus horrible de la barbarie humaine et du Mal à l'état pur? Espoir de rédemption par une espèce de baiser au lépreux ? Désir profond de partager la souffrance humaine? Britten parle peu de cette expérience terrifiante, pas même à Menuhin, mais l'une des rescapées du camp devient plus tard musicienne au sein de The English Chamber Orchestra qui assure la création de plusieurs de ses œuvres à partir de 1960, et en ravive le souvenir. À la fin de sa vie il confie à Peter Pears combien l'expérience avait coloré tout ce qu'il avait écrit par la suite, notamment les bouleversants Holy Sonnets of John Donne, op. 35, et son opéra The Rape of Lucretia, créés après le retour des camps de la mort. C'est le premier des opéras où il met en scène l'ubiquité du Mal, et le scandale de son existence dans les œuvres parfaites de la Création, comme dans Billy Budd, dont l'univers concentrationnaire et la discipline aveugle rappellent celle de camps, et dans Le tour d'écrou d'après Henry James en 1954, où l'innocence même des victimes est cause de leur destruction.

\section{Un pacifiste à la mode}

L'expérience ne remet pas en cause ce pacifisme épidermique mais en 1952 son ami le critique Hans Keller postule que cette violence, qui s'exprime notamment dans le traitement des percussions et dans les «Dances of Death » précédemment indiquées, n'est que la traduction de la répression du sadisme qui sous-tend tout pacifisme ${ }^{23}$. On lui laisse la responsabilité de cette analyse. Britten et Pears trouvent de nombreuses occasions d'exprimer ce pacifisme en ce siècle que troublent les deux Guerres mondiales, la Guerre Froide, la menace nucléaire et la guerre du Vietnam. Le programme d'un de leur récitals à New York, pendant leur grande tournée au Canada et aux États-Unis entre octobre et décembre 1949, inclut une déclaration contre la "guerre moderne » qui, avec les armes atomiques et bactériologiques, est devenue irrationnelle, suicidaire et immorale. En plein McCarthysme, le résultat ne se fait pas attendre. Dès février 1942, Britten et Pears avaient attiré l'attention du directeur du FBI, J. Edgar Hoover, sans doute à cause des idées de gauche défendues dans Paul Bunyan, dont les Vieux Arbres conservateurs du Prologue traitent les Jeunes Arbres de « Rouges ». En 1949, Britten et Pears deviennent « prohibited immigrants ", les obligeant à effectuer une demande de visa contraignante et humiliante pour voyager aux Etats-Unis. La mesure ne sera levée qu'en 1983, après la mort de Britten. 
21 Si le War Requiem constitue la manifestation la plus éclatante de ce pacifisme en 1962, et s'il trouve un si large écho dans le public, c'est que les attitudes vis-à-vis de la guerre aussi ont changé. Ainsi les poètes comme Wilfred Owen et Siegfried Sassoon, rejetés par la majorité du public dans les années Vingt pour insulte à la patrie, sont officiellement reconnus et mis au programme des A levels dans les années Soixante et le pacifisme de Britten devient alors consensuel. En mars 1965 Britten est décoré de l'Ordre du Mérite, distinction limitée à vingt-quatre récipiendaires vivants, conférée par la Reine Elizabeth II en personne, qui est présente à l'inauguration du complexe de Maltings de Snape, où se déplace en 1967 le festival d'Aldeburgh. C'est la plus prestigieuse des décorations que Britten ait reçue du Royaume-Uni avant la pairie : il devint Lord Britten six mois avant sa mort, en décembre 1976.

Si Britten participe à des tournées de concerts organisées sous l'égide du British Council pour promouvoir la culture et la musique anglaise et contenir l'influence du communisme (comme en témoignent son long voyage en Extrême-Orient en 1956 ou son premier séjour en URSS en 1963), son pacifisme s'exprime dans des œuvres personnelles avant ou après 1960. Ainsi le Canticle III, Still Falls the Rain, de 1954, sur un poème d'Edith Sitwell, soustitré The Raids 1940, Night and Dawn, évoque Londres sous les bombes, l'humanité souffrante et la passion du Christ, comme le désespoir de son ami le pianiste Noel Mewton Wood qui se suicide après la mort de son compagnon. De même, les huit poèmes du Nocturne pour ténor, sept instruments et cordes, op. 60, de 1958, comprennent la méditation nocturne de Wordsworth sur les massacres de septembre extraite du livre X de The Prelude, suivie directement de l'évocation de la Mort et des cadavres de soldats pétrifiés dans le poème The Kind Ghosts de Wilfred Owen. Malgré son caractère de commande officiel, le War Requiem est aussi une œuvre personnelle, par le choix des poèmes d'Owen et de ses trois interprètes représentant les pays engagés dans la Deuxième Guerre mondiale: le Britannique Peter Pears, l'Allemand Dietrich FischerDieskau et la Russe Galina Vischnesvkaia ${ }^{24}$. Épouse du violoncelliste Mstislav Rostropovitch, pour lequel Britten écrit pas moins de cinq œuvres, les deux Russes deviennent des amis chez qui Britten et Pears font plusieurs séjours entre 1963 et 1971, pendant le « Dégel » des années Khrouchtchev et la répression qui s'ensuit; à l'issue de celle-ci, Britten accueille le couple chez lui en 1974 lorsque mari et femme sont déchus de leur nationalité pour "agissements antipatriotiques". En 1960, grâce à Slava Rostropovitch, Britten rencontre Dimitri Chostakovitch qu'il admire depuis les années Trente et avec qui il noue une touchante amitié25.

Le War Requiem est suivi d'une commande qui dépasse le cercle du Royaume-Uni puisqu'elle émane de la Croix Rouge pour la célébration de son centenaire. Britten choisit de mettre en musique la parabole du Bon Samaritain, sur un texte latin de Patrick Wilkinson, dans cette Cantata Misericordium, op. 69 de 1963, qui exalte l'esprit de compassion et semble être un écho apaisé du War Requiem. Enfin l'hymne Voices for Today, op.75, de 1965 est le résultat d'une commande pour fêter les vingt ans de l'ONU et rassemble des citations du Christ, de Sophocle, de Virgile, de Blake et de Camus. Jouée simultanément à Londres, Paris et New York c'est une des dernières grandes commandes faites à Britten au plan international ou, du moins, la dernière qu'il honore, se concentrant alors sur des thèmes qui le touchent plus particulièrement. 


\section{Enfants sacrifiés et pères assassins}

24 grande empathie envers eux, s'emploie à défendre leur cause en composant pour eux : ne décide-t-il pas d'adopter un petit Basque, Antoni Barrutia, réfugié de la Guerre d'Espagne en $1938^{26}$ ? Parallèlement à son pacifisme, il revient constamment au thème de l'enfance humiliée, meurtrie, ou sacrifiée telle qu'il la présente dans The Little Sweep de 1949, œuvre inspirée des souffrances des petits ramoneurs de William Blake. Seconde partie d'un diptyque éducatif et récréatif appelé Let's Make an Opera, qui raconte comme se crée un opéra, il inclut non seulement des enfants sur scène mais fait chanter le public et les enfants dans la salle, en écho au Second Hurricane de Copland, alors que s'ébauche en Angleterre la pédagogie centrée sur l'enfant. Le procédé est repris dans Noye's Fludde de 1958, où aux adultes chanteurs et musiciens se mêlent les enfants chanteurs et instrumentistes, ainsi que le public. En 1965, pour remercier Dietrich Fischer-Dieskau de sa prise de rôle dans le War Requiem et la Cantata Misericordium, Britten et Pears élaborent pour lui un cycle chant et piano créé au festival d'Aldeburgh, intitulé Songs and Proverbs of William Blake, op. 74, qui puise dans Songs of Experience, Auguries of Innocence, et Proverbs of Hell. Pears choisit les poèmes les plus noirs, les plus violents et les plus révoltés de Blake, London, The Chimney Sweeper, A Poison Tree et The Tyger, qui mêlent des images de l'enfance torturée au nom de grands principes, «the mind-forged manacles », et des manifestations du Mal. Il fait dire au critique John Warrack que Britten y confronte ce qu'il y a de plus sombre et de plus cruel chez lui, et qui hante toujours son art, analyse qui rejoint celle Hans Keller citée plus haut ${ }^{27}$. Il est vrai que certaines œuvres de Britten mettent en scène son propre théâtre de la cruauté, qui rappelle une certaine tragédie élisabéthaine par son déchaînement de violence. The Golden Vanity, composée en 1966, raconte la traitrise d'un capitaine de vaisseau refusant la main de sa fille promise au jeune mousse qui a sauvé son navire au péril de sa vie. En 1967, Britten envisage un opéra fondé sur la nouvelle de Mérimée Matéo Falcone, où un jeune garçon est exécuté par son père pour avoir trahi un proscrit malgré luii ${ }^{28}$. En mai 1969 le chœur d'enfants de l'école de Wandsworth, pressenti pour chanter la ballade des Wingrave dans l'opéra pacifiste Owen Wingrave de 1971, crée à Londres The Children's Crusade, sur un texte de Brecht, pour le cinquantième anniversaire de Save The Children Fund, organisation caritative fondée en Grande-Bretagne en 1919 dans le but de venir en aide aux enfants en détresse dans le monde. Le choix du poème est inspiré à Britten par le souvenir d'une représentation de Mère Courage, avec Theresa Giehse dans le rôle-titre, pièce qu'il voit après la guerre à Zurich, et qui n'a rien de festif. Accompagné de percussions et de deux pianos eux aussi percussifs, il décrit l'errance d'un groupe d'enfants polonais dans la tourmente de l'hiver 1940, l'échec de leur petite société et leur mort pitoyable de froid et de faim. La même année, son pendant intime, le cycle Who are these children ?, composé sur des poèmes de William Soutar, confronte des enfants, les pieds nus dans des ruines après un raid, et des aristocrates en uniforme rouge, échappés d'une chasse à courre. L'Idoménée, le seul opéra de Mozart que Britten ait monté, met en scène un père prêt à sacrifier son fils dans un épisode qui se situe après la guerre de Troie. Donné au festival d'Aldeburgh en juin 1969, enregistré par BBC TV en septembre 1969, il est repris en juin 1970.

enfants sacrifiés par des pères ou des adultes assassins, déjà mis en scène dans le Tour d'écrou de 1954, constitue aussi la trame de l'opéra pacifiste Owen Wingrave de 
1971. Le livret est fondé sur un récit fantastique de Henry James, que Britten lit dans les années Trente et dont le souvenir lui revient avec force pendant la composition du War Requiem. Il met en scène le jeune Owen, dernier rejeton d'une famille de tradition militaire, qui la rejette, les efforts de sa famille pour le remettre dans le droit chemin et la mort d'Owen dans le salon rond, véritable antre du Mal. Y sont morts un lointain jeune ancêtre aux mains de son père, lui-même retrouvé mort dans ce salon, et condamnés depuis à hanter le domaine de Paramore. Une fois encore Britten est à l'écoute de son époque puisque son travail sur Wingrave s'inscrit entre l'invasion de la Tchéquoslovaquie par les chars soviétiques en 1968 et la fusillade de la police sur les manifestants protestant contre l'engagement américain au Cambodge à l'université de Kent State en mai 1970. Britten, curieux de toute innovation technique ${ }^{29}$, et profitant de toutes les occasions pour faire avancer la cause de l'opéra, malgré les réserves exprimées dans son discours d'acceptation du Prix Aspen, compose l'œuvre expressément pour la télévision, dont la diffusion se fera simultanément dans douze pays.

La diffusion de Wingrave en mai 1971 est précédée d'une campagne de publicité sans précédent pour souligner l'unicité de cette création ${ }^{30}$. Après le succès planétaire du War Requiem, producteurs et réalisateurs de télévision, encore sensibles aux idéaux de John Reith, assaillent Britten de propositions. Les années Soixante sont une période de grande expérimentation pour BBC TV, qui s'emploie à servir son compositeur national. En 1963, le réalisateur Humphrey Barton célèbre les 50 ans de Britten avec un documentaire spécial, Britten at Fifty. En 1964, BBC TV enregistre un récital de Britten et de Pears. Le succès fait au Billy Budd, filmé par la BBC en 1966, entraîne d'autres réalisations, comme un documentaire TV, Benjamin Britten et son festival, tourné pendant le festival de juin et diffusé en novembre 1967, L'occasion en est l'ouverture de la salle de Maltings de Snape, mentionnée plus haut, qui permet enfin à Britten de disposer de la salle de ses rêves et de fuir les studios de Londres pour travailler chez lui, d'où l'enregistrement de Peter Grimes par BBC TV en 1968. La télévision offre à Britten son petit écran qui lui permet d'y projeter sa subjectivité, poursuivant ainsi les leçons de Grierson au GPO Film Unit.

$\mathrm{Au}$ final, Britten est très déçu de la version télévisée de son opéra et s'empresse de « détéléviser » Wingrave pour sa création à Covent Garden en mai $1973^{31}$. Pour autant, il est très conscient que la télévision lui offre un forum international pour exprimer des sentiments personnels : «Owen Wingrave semble avoir créé un impact - espérons aussi que les gens ont bien compris le message de l'histoire ${ }^{32}$ ! » écrit-il à son ami William Plomer. Lorsque Britten parle de son opéra comme de l'explosion d'une bombe au sein de la famille Wingrave, il sait de quoi il parle, puisque sa vie est ponctuée d'incidents douloureux dus à son pacifisme et son homosexualité, l'un fonctionnant parfois comme un paravent pour l'autre. L'air de la paix d'Owen à l'acte II, aux résonances whitmaniennes, peut se lire comme l'acceptation d'une différence, et la révolte de la fin de l'air comme une protestation contre les lois victoriennes, alors que le Rapport Wolfenden, qui vise à décriminaliser l'homosexualité entre adultes consentants, paru en 1957, donne enfin forme, en 1967, la loi qui abroge celle votée après le procès d'Oscar Wilde.

\section{Conclusion}

Comme bien souvent chez Britten, une œuvre assortie de gros moyens financiers est suivie d'une autre plus intime. En septembre 1970, la partition de Wingrave à peine 
terminée, Britten reprend contact avec Golo Mann pour lui faire part de son projet d'adapter la nouvelle de son père La mort à Venise. Golo accepte tout en remarquant que son père en 1948 disait que si l'on tirait un film de son Doktor Faustus, il aimerait que Britten en écrive la musique. Britten, Pears et Myfanwy Piper sa librettiste, élaborent un premier scénario pour leur nouvel opéra fin janvier 1971. Sa santé déclinant et Pears vieillissant, Britten a conscience que cet opéra, qui renverse la situation de Billy Budd et du Tour d'écrou puisque c'est l'homme âgé qui devient la victime du garçon dont il tombe amoureux, sera le dernier. Le travail de révision de Wingrave pour la création à Covent Garden se fait parallèlement à la composition de La mort à Venise, programmée pour le vingt-sixième festival d'Aldeburgh. Les deux œuvres sont créées à six semaines d'intervalle, en mai et en juin 1973, mais entre-temps Britten a subi une opération du cœur différée depuis longtemps et ne peut y assister. Sa Mort à Venise remporte un grand succès à Londres en octobre 1973 et au Met newyorkais en 1974. Parabole sur l'inspiration artistique, c'est aussi le dernier volet d'une série d'œuvres, commencée avec Peter Grimes, qui mettent en scène les rapports ambigus entre enfants et adultes et les tourments de l'homosexualité, et qui posent la question de la place de l'artiste dans la société de son temps. L'acceptation de la pairie au nom de tous les compositeurs britanniques qui l'ont précédé est aussi pour Britten la reconnaissance de son engagement pour la musique et du droit enfin accordé à sa différence.

\section{BIBLIOGRAPHIE}

BRITTEN Tony, Benjamin Britten : Peace and Conflict, Capriol Film Productions, 2013.

CARPENTER Humphrey, Benjamin Britten, A Biography, Londres, Faber, 1992.

CARPENTER Humphrey, W. H. Auden, A Biography, Oxford, Oxford University Press, 1981.

COOKE Mervyn (dir.), The Cambridge Companion to Britten, Cambridge, Cambridge University Press 1999.

COUDERC Gilles, «Britten et les Russes : itinéraires croisés », Accords Majeurs, les échanges musicaux entre la Russie et le monde (XIXe-XXe siècles), Slavica Occitania, $\mathrm{n}^{\circ} 23$, Toulouse, 2007.

van GYSEGHEM André, «British Theatre in the Thirties : An Autobiographical Record », in CARK Jon, HEINEMAN Margot, MARGOLIES David, SNEE Carol (dir.), Culture and Crisis in Britain, Londres, Lawrence \& Wishart, 1979.

HARDY Henry Forsyth, John Grierson : A Documentary Biography, Londres, Faber, 1979.

HYNES Samuel, The Auden Generation, Literature and Politics in England in the Thirties, Londres, The Bodley Head, 1976, Pimlico, 1992.

KELLER Hans, «The Musical Character », in MITCHELL Donald et KELLER Hans (dir.), Benjamin Britten, A Commentary on his Works from a Group of Specialists, Londres, Rockcliff, 1952.

KILDEA Paul (dir.), Britten on Music, Oxford, Oxford University Press, 2003.

KILDEA Paul, Selling Britten, Oxford, Oxford University Press, 2003 
KILDEA Paul, « Britten, Auden and "Otherness" », in COOKE Mervyn (dir.), The Cambridge Companion to Britten, Cambridge, Cambridge University Press, 1999.

MITCHELL, Donald. Britten \& Auden in the Thirties, Londres, Faber, 1981, Woodbridge, The Boydell Press, 2000.

MITCHELL Donald et REED Philip (dir.), Letters from a Life, Selected Letters and Diaries of Benjamin Britten, Volume I, 1923-1939; Volume II, 1939-1945, Londres, Faber, 1990.

MORRIS Peter, «Re-thinking Grierson: The Ideology of John Grierson », in O’REGAN Tom et SHOESMITH Brian (dir.), History on/and/in Film, Perth, History \& Film Association of Australia, 1987.

O’REGAN Tom et SHOESMITH Brian (dir.), History on/and/ in Film, Perth, History \& Film Association of Australia, 1987.

REED Philip et COOKE Mervyn, Letters from a Life, Selected Letters of Benjamin Britten, Volume VI, 1966-1976, Woodbridge, The Boydell Press in association with the Britten-Pears Foundation, 2012.

RUPPRECHT Philip (dir.), Rethinking Britten, Oxford, Oxford University Press, 2013.

SINFIELD Alan, Literature, Politics, and Culture in Postwar Britain, Berkeley et Los Angeles, University of California Press, 1989.

SPENDER Stephen, World within World, The Autobiography of Stephen Spender, Londres, Faber, 1951.

WARD-GRIFFIN Danielle, « Animating Owen Wingrave », in RUPPRECHT Philip (dir.), Rethinking Britten, Oxford, Oxford University Press, 2013.

\section{NOTES}

1. «Je crois que tout artiste doit avoir conscience d'être un être humain. Il fait partie de la société et ne devrait pas s'enfermer dans une tour d'ivoire. Je pense qu'il a des devoirs à accomplir vis-àvis de ses frères humains. Ce n'est pas seulement une obligation; je crois que c'est aussi un plaisir. Je veux que l'on joue ma musique. Je préfèrerais-peut être que cela vous semblera stupide de le dire-mais je préfèrerais que l'on joue ma musique plutôt que composer des chefs d'œuvre qui ne soient pas joués.» KILDEA Paul (dir.), Britten on Music, Oxford, Oxford University Press, 2003, p. 311-312.

2. «À Benjamin Britten, , remarquable compositeur, concertiste et interprète, qui a vraiment inspiré l'humanité pour parvenir à une plus grande compréhension, clarification et acceptation de sa nature, de ses motivations et de sa destinée particulière, grâce à la musique, évocatrice des sentiments, des humeurs et des pensées de l'être humain. »

3. «La raison essentielle à mon opposition au fascisme, c'est qu'à l'école, j'ai vécu dans un état fasciste. " KILDEA Paul, Britten, Auden and 'Otherness', in COOKE Mervyn (dir.), The Cambridge Companion to Britten, Cambridge, Cambridge University Press, 1999, p. 42

4. «[Les enfants] me trahissaient par obéissance à l'esprit de l'école. La grande perfidie caractéristique des enfants contaminait toute l'école, comme dans un état fasciste où chacun se rend compte, même s'il n'en a pas conscience lui-même, que son voisin est un agent voué aux intérêts de la police. " Stephen SPENDER, World within World, The Autobiography of Stephen Spender, Londres, Faber, 1951, p. 329.

5. BRITTEN Tony, Benjamin Britten : Peace and Conflict, Capriol Film Productions, 2013.

6. MITCHELL Donald et REED Philip (dir.), Letters from a Life, The Selected Letters and Diaries of Benjamin Britten, Volume II, 1939-45 (LFL vol. II), Londres, Faber, 1990, p.1049. 
7. MORRIS Peter, «Re-thinking Grierson: The Ideology of John Grierson », in O'REGAN Tom et SHOESMITH Brian (dir.), History on/and/ in Film, Perth, History \& Film Association of Australia, 1987, p. 20-30. HARDY Henry Forsyth, John Grierson : A Documentary Biography, Londres, Faber, 1979.

8. MITCHELl Donald, Britten \& Auden in the Thirties, Londres, Faber, 1981, Woodbridge, The Boydell Press, 2000. CARPENTER Humphrey, Benjamin Britten, A Biography, Londres, Faber, 1992. CARPENTER Humphrey, W. H. Auden, A Biography, Oxford: Oxford University Press, 1981.

9. «Je pense que vous aimeriez beaucoup WHA. C'est une personnalité étonnante, mais absolument sincère et très brillante. Il possède une culture immense, non seulement littéraire, mais elle embrasse tout ce qui concerne les arts, et la politique en particulier, et sur ce point, il va dans la direction qui, je ne puis m'empêcher de le penser, est celle où doit aller toute personne sérieuse et tout artiste en particulier, la lutte contre le fascisme qui, c'est évident, restreint toute liberté de pensée. » MITCHELL Donald et REED Philip (dir.), Letters from a Life, The Selected Letters and Diaries of Benjamin Britten, Volume I, 1923-1939, (LFL vol. I), Londres, Faber, 1990, p. 378. La grammaire est conforme à l'original.

10. «Mais le rôle de la poésie n'est pas de nous dire ce qu'il faut faire mais d'étendre notre connaissance du bien et du mal, rendant peut-être la nécessité d'agir plus urgente et sa nature plus évidente, mais nous conduisant seulement au point où il nous devient possible de faire un choix rationnel et moral. » HYNES Samuel, The Auden Generation, Literature and Politics in England in the Thirties, Londres, The Bodley Head, 1976, Pimlico, 1992, p. 14.

11. "Il faudra toujours qu'il y deux sortes d'art, l'art-évasion, car l'homme a besoin de s'évader autant que de nourriture et de sommeil profond, et l'art-parabole, cet art qui enseignera à l'homme à désapprendre la haine et à apprendre l'amour. » HYNES Samuel, The Auden Generation, op.cit., p.14

12. «Quasiment le seul écrivain britannique d'envergure qui écrivait alors pour le nouveau théâtre réaliste porteur d'une conscience sociale et des intérêts de la classe ouvrière. » van GYSEGHEM André, "British Theatre in the Thirties : An Autobiographical Record », in CARK Jon, HEINEMAN Margot, MARGoliEs David et SNEE Carol (dir.), Culture and Crisis in Britain, Londres, Lawrence \& Wishart, 1979.

13. LFL, vol. I, p 477.

14. LFL, vol. II, p. 1334.

15. LFL, vol. II, p. 567.

16. LFL, vo. II, p. 635.

17. Britten oublie Bunyan jusqu'en 1973 où, suite à de graves problèmes de santé et à la perte de l'inspiration, il est convaincu par son ami Donald Mitchell de réviser des œuvres anciennes non publiées. Des extraits de Bunyan sont donnés avec succès au festival d'Aldeburgh de 1974, un an après la mort d'Auden, encourageant Britten à poursuivre ses révisions. L'œuvre complète est présentée en concert par la BBC en février 1976 puis donnée au festival d'Aldeburgh de 1976, six mois avant la mort de Britten.

18. LFL, vol. II, p.869.

19. $L F L$, vol. II, p. 869-873 et 957-959.

20. «Je fais de [ma symphonie] une œuvre aussi pacifiste que possible. Je ne crois pas qu'on puisse transmettre des théories sociales ou politiques ou économiques en musique, mais en associant de la nouvelle musique avec des motifs musicaux célèbres, je crois qu'on peut faire passer certaines idées. On prend le risque de la confusion à discuter de telles choses. Tout ce dont je suis sûr, alors que je la compose, c'est de mes convictions pacifistes. » CARPENTER Humphrey, Benjamin Britten, A Biography, op. cit., p. 146.

21. «Puisque je crois que l'esprit de Dieu réside dans chaque homme, je ne peux pas détruire des vies humaines, et c'est mon devoir, je le crois, d'éviter de contribuer à le faire autant que je le peux, si fort mon rejet des actions ou les pensées d'un être humain soit-il. Ma vie entière a été 
consacrée à des actes de création [...] et je ne peux prendre part à des actes de destruction [...] Je crois sincèrement que le meilleur moyen d'aider mes frères humains est de continuer le travail pour lequel je suis le plus qualifié par la nature de mes dons et par mes études, c'est à dire la création ou la diffusion de la musique. » LFL, vol. II, p. 1046.

22. SINFIELD Alan, Literature, Politics, and Culture in Postwar Britain, Berkeley et Los Angeles, University of California Press, 1989.

23. KELleR Hans, "The Musical Character », in MITCHELl Donald et KELLER Hans (dir.), Benjamin Britten, A Commentary on his Works from a Group of Specialists, Londres, Rockcliff, 1952, p. 327 et 350.

24. Elle participe à l'enregistrement de l'œuvre dirigée par Britten mais il lui est interdit d'être présente à sa création, la Ministre de la Culture soviétique refusant sa présence dans une cathédrale à côté d'un Allemand.

25. COUDERC Gilles, "Britten et les Russes: itinéraires croisés ", Accords Majeurs, les échanges musicaux entre la Russie et le monde (XIXe-XXe siècles), Slavica Occitania, $\mathrm{n}^{\circ} 23$, Toulouse, 2007, p. 91-106.

26. CARPENTER Humphrey, Benjamin Britten, A Biography, op cit., p. 116-117.

27. CARPENTER Humphrey, Benjamin Britten, A Biography, op. cit., p.449.

28. REED Philip et COOKE Mervyn (dir.), Letters from a Life, The Selected Letters of Benjamin Britten, Volume VI, 1966-1976 (LFL), Woodbridge, The Boydell Press in association with the Britten-Pears Foundation, 2012, p. 114.

29. KILDEA Paul, Selling Britten, Oxford, Oxford University Press, 2003.

30. WARD-GRIFFIN Danielle, "Animating Owen Wingrave ", in RUPPRECHT Philip (dir.), Rethinking Britten, Oxford, Oxford University Press, 2013.

31. LFL vol. VI, p, 428 et 542.

32. LFL, vol. VI, p. 428.

\section{RÉSUMÉS}

Compositeur, chef d'une troupe d'opéra, directeur d'un festival de musique, chef d'orchestre et pianiste accompagnateur, Britten n'est pas seulement engagé à défendre son art mais un art au service de ses contemporains. L'idée de cet engagement lui vient de son compagnonnage avec le poète W.H. Auden dans les années 1935-1939 et de son travail au sein du GPO Film Unit avec John Grierson, et par l'adoption de "l'art de la parabole » défini par le poète. Cet engagement se manifeste dans la création d'œuvres "personnelles " où Britten exprime sa différence de pacifiste en temps de guerre et d'homosexuel condamné au secret, comme dans celles de commandes officielles, de la Croix-Rouge ou des Nations-Unies, qui font de lui un «musicien de circonstance ». Il s'exprime aussi dans la composition d'œuvres destinées aux enfants en tant qu'interprètes ou impliquant la participation du public et d'autres où il traduit son questionnement sur la place de l'artiste dans la société de son temps.

A composer, the leader of an opera group, the director of a festival, a conductor and concert pianist, Benjamin Britten (1913-1976) was not only engaged in defending his craft but also in being an artist at the service of his community. This commitment sprang from his collaboration with the poet W.H. Auden in the mid-Thirties, whose concept of "parable art" he borrowed; and with John Grierson, the inventor of the documentary, at the GPO Film Unit. His engagement 
dictated personal works in which Britten expressed his peculiarity as a pacifist in war time and as a homosexual condemned to silence, as well as more official works commissioned by great institutions like the Red Cross or the UN which made of him "a musician for an occasion". His commitment also resulted in compositions for children, performed by children, and in works that questioned the place of the artist in society.

INDEX

Mots-clés : pacifisme, homosexualité, W.H. Auden, John Grierson, art de la parabole, Britten Keywords : pacifism, homosexuality, W.H. Auden, John Grierson, parable-art, Britten

\section{AUTEUR}

\section{GILLES COUDERC}

Université de Caen, équipe ERIBIA 